\title{
Caracterização reológica de sorvetes
}

\author{
Rheological characterization of ice cream
}

\author{
Katherine Helena OLIVEIRA ${ }^{1}$, José Antonio Ribeiro de SOUZA ${ }^{1}$, Alcilene Rodrigues MONTEIRO ${ }^{1 *}$
}

\begin{abstract}
Resumo
O conhecimento do comportamento reológico é de grande importância no processamento, manuseio, controle de qualidade e desenvolvimento de produtos alimentícios. A literatura relata que a maioria dos alimentos apresenta comportamento não newtoniano, em que a sua viscosidade pode variar com o tempo e com as condições de escoamento. Neste trabalho foi realizada a caracterização reológica de cinco diferentes tipos de sorvetes. As amostras nos sabores creme, creme light, limão, iogurte e soja banana foram fornecidas pela empresa Amoratto Sorvetes Artesanais, localizada na cidade de Florianópolis/SC. Os dados reológicos foram obtidos através de um reômetro com geometria de cilindros concêntricos. Os ensaios foram realizados em três diferentes temperaturas $\left(-2,0\right.$ e $\left.2{ }^{\circ} \mathrm{C}\right)$ e os resultados experimentais foram ajustados pelos modelos de Bingham, Casson, Herschel-Bulkley e Lei da Potência. As diferentes amostras apresentaram comportamento não newtoniano nas três temperaturas. Os parâmetros reológicos $(K \mathrm{e} n)$ foram obtidos através do ajuste dos modelos e o da Lei da Potência mostrou o melhor ajuste aos resultados, com coeficientes de correlação $(r)$ iguais a 0,99 para quatro das cinco amostras, nas três diferentes temperaturas.

Palavras-chave: reologia; sorvete; viscosidade.
\end{abstract}

\begin{abstract}
The knowledge of rheological behavior is an important part of processing, handling, quality control and development of food products. Literature shows that most foodstuffs present non-Newtonian behavior, where their viscosity may vary with time, as well as with flow conditions. The rheological characterization of five different kinds of ice cream was carried out in this work. The samples studied were vanilla flavor, light vanilla flavor, lemon, yoghurt and banana soy, supplied by Amoratto Sorvetes Artesanais, located in Florianópolis/SC, Brazil. The rheological data were obtained using a rotational viscosimeter with concentric cylinder geometry. The trials were carried out at three different temperatures $\left(-2,0\right.$ and $\left.2{ }^{\circ} \mathrm{C}\right)$ and experimental results were fitted by Bingham, Casson, Herschel-Bulkley and Power Law models. The different samples showed non-Newtonian behavior at the three temperatures. The rheological parameters $(k$ and $n)$ were obtained through fitting of the models and the Power Law model presented the best results, with $r=0.99$ for four of the five samples, at the three different temperatures.
\end{abstract}

Keywords: rheology; ice cream; viscosity.

\section{Introdução}

O sorvete à base de leite pode ser considerado como uma suspensão aerada de gordura e cristais de gelo numa solução concentrada de açúcar que contém hidrocolóides, gordura e proteínas. A composição do sorvete à base de leite pode variar de acordo com a região ou o local onde é produzido. A variação na sua composição é de 8 a $20 \%$ de gordura, 8 a $15 \%$ de sólidos não gordurosos do leite, 13 a $20 \%$ de açúcar e 0 a $0,7 \%$ de emulsificante-estabilizante (ARBUCKLE, 1977). A composição do sorvete interfere nas suas características físicas porque está relacionada com o processo, que influenciará diretamente o estado de agregação dos glóbulos de gordura, a quantidade de ar incorporada, o tamanho das bolhas de ar, a viscosidade da fase aquosa e o tamanho e estado de agregação dos cristais de gelo (DICKINSON; STAINSBY, 1982). A viscosidade da mistura é afetada pela composição (principalmente gordura e estabilizante), tipo e qualidade dos ingredientes, processamento e manuseio da mistura. Com o aumento da viscosidade, a resistência ao derretimento e a cremosidade do sorvete aumentam. Então, a viscosidade desejada pode ser assegurada controlando a composição da mistura (ARBUCKLE, 1977).
O conhecimento do comportamento reológico dos alimentos é útil não somente para o controle de qualidade do produto, mas principalmente para o dimensionamento de sistemas de tubulação, trocadores de calor, filtros, bombas, entre outros (VASQUES, 2003). O comportamento dos fluidos é descrito através de modelos reológicos, que relacionam tensão de cisalhamento com a taxa de deformação.

O modelo reológico mais simples é o newtoniano, que apresenta uma relação linear entre tensão de cisalhamento e taxa de deformação. No entanto, a maioria dos alimentos fluidos não apresenta esse tipo de comportamento e requer modelos mais complexos para sua caracterização (HOLDSWORTH, 1971; TABILO-MUNIZAGA; BARBOSA-CÁNOVAS, 2005). A escolha do modelo a ser utilizado é uma função das características do fluido (RAO; ANANTHESWARAN, 1982). Os modelos mais comumente utilizados são: Ostwald-de Waele, Plástico de Bingham, Hershel-Bulkley e Casson.

O comportamento reológico de sorvetes produzidos à base de leite foi estudado por diversos autores como Aime et al.

Recebido para publicação em 19/3/2007

Aceito para publicação em 4/3/2008 (002394)

${ }^{1}$ Laboratório de Propriedades Físicas, Departamento de Engenharia Química e Engenharia de Alimentos, Universidade Federal de Santa Catarina - UFSC,

Campus Trindade, CP 476, CEP 88040-900, Florianópolis - SC, Brasil, E-mail: alcilene@enq.ufsc.br

${ }^{*}$ A quem a correspondência deve ser enviada 
(2001); Kaya e Tekin (2001); Cotrel, Geofrey e Phillips (1980); e Smith, Bakshi e Lomauro (1984) e mostraram comportamento não newtoniano. As propriedades reológicas da maioria dos sorvetes têm sido descritas como pseudoplásticas (COTREL; GEOFREY; PHILLIPS, 1980; GOFF; DAVIDSON, 1992). Porém, sorvetes produzidos a partir de outras bases não têm sido pesquisados.

O objetivo deste trabalho foi estudar as propriedades reológicas de cinco diferentes tipos de sorvete, em três temperaturas distintas, e caracterizar o seu comportamento através do ajuste de modelos reológicos.

\section{Material e métodos}

\subsection{Amostras}

As amostras de sorvetes foram fornecidas pela empresa Amoratto Sorvetes Artesanais, localizada na cidade de Florianópolis, estado de Santa Catarina, Brasil.

Os diferentes tipos de sorvetes utilizados para os ensaios reológicos foram: Linha Base Leite, Linha Light, Linha Base Água, Linha Frozen e Linha Soya. Uma amostra foi escolhida para representar cada um dos diferentes tipos. As amostras representantes e a respectiva linha estão descritas na Tabela 1.

Para cada tipo de sorvete foram realizadas análises para determinar o teor de gordura total e o teor de gorduras saturadas, carboidratos, proteínas, colesterol, cálcio, sódio e fibra alimentar, conforme normas do Instituto Adolfo Lutz (1997).

\subsection{Processo de obtenção do sorvete}

A Figura 1 apresenta um fluxograma das etapas do processo de fabricação utilizado na produção dos sorvetes.

Preparação da calda: os ingredientes secos e líquidos foram adicionados no pasteurizador durante o processo de aquecimento, sob agitação contínua.

Pasteurização: a calda foi pasteurizada a $80{ }^{\circ} \mathrm{C}$ por 25 segundos em trocador de calor a placas.

Homogeneização: no processo de homogeneização a pressão utilizada variou conforme o tipo de sorvete. Para os sorvetes de creme, creme light e iogurte, a pressão foi $100 \mathrm{kgf.cm}{ }^{-2}$ e $80 \mathrm{kgf} . \mathrm{cm}^{-2}$, respectivamente. A pressão média utilizada na homogeneização dos sorvetes de soja banana e limão foi 75 kgf. $\mathrm{cm}^{-2}$.

Maturação: a calda resfriada foi transferida para as tinas de maturação, onde permaneceu sob agitação lenta, a uma temperatura de $4{ }^{\circ} \mathrm{C}$, por um tempo mínimo de 2 horas. De

Tabela 1. Amostras de sorvete utilizadas neste trabalho.

\begin{tabular}{lc}
\hline \multicolumn{1}{c}{ Amostras estudadas } & Linha comercial \\
\hline Sorvete de creme de Baunilha & Base Leite \\
Sorvete de creme de Baunilha Light & Light \\
Sorvete de Limão & Base água \\
Sorvete de soja de Banana & Soya \\
Sorvete de iogurte com Amarena & Frozen \\
\hline
\end{tabular}

acordo com cada tipo de sorvete, foi feita a adição de suco de fruta e/ou saborizante.

Congelamento e incorporação de ar: A calda maturada foi batida e congelada em um processo contínuo, à temperatura de $-8{ }^{\circ} \mathrm{C}$. A incorporação de ar (overrun) do sorvete variou dependendo do tipo de sorvete: creme $80 \%$ de ar incorporado, creme light $100 \%$, limão $80 \%$, soja banana $80 \%$ e iogurte $70 \%$ de ar. Nesta etapa, cerca de $50 \%$ da água foi congelada.

Envase: na saída da produtora, o sorvete foi acondicionado em embalagens plásticas de polipropileno injetado com capacidade de $2 \mathrm{~L}$, com exceção do sorvete de soja banana, que foi embalado em potes plásticos com capacidade de $1 \mathrm{~L}$.

Endurecimento: o sorvete envasado foi levado para uma câmara frigorífica, com temperatura de $-22^{\circ} \mathrm{C}$, onde continuou o seu processo de congelamento. Com o endurecimento, cerca de $90 \%$ da água foi congelada.

\subsection{Caracterização reológica do sorvete}

\section{Descrição do equipamento}

O comportamento reológico das amostras foi determinado utilizando um viscosímetro rotacional (Thermohaake, modelo VT550, Paramus, EUA), com geometria de cilindros concêntricos (MV/MV1), disponível no Laboratório de Materiais da Universidade Federal de Santa Catarina. O viscosímetro possui um banho termostático que permite o controle da temperatura da amostra durante o ensaio. Os ensaios para as medidas reológicas foram realizados a $-2,0$ e $2{ }^{\circ} \mathrm{C}$. Para o abaixamento da temperatura, adicionou-se etilenoglicol na água de refrigeração contida no banho termostático. O equipamento

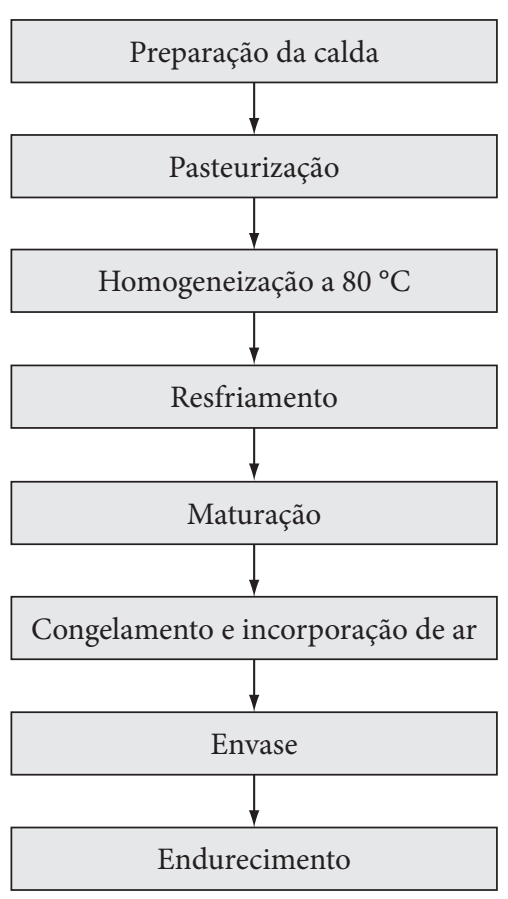

Figura 1. Fluxograma do processo de fabricação do sorvete. 
possui um sistema de aquisição de dados que permite obter os valores de taxa de deformação e tensão de cisalhamento, através do software (Haake Rheowin 3, Paramus, EUA) que ajusta os dados experimentais.

\section{Medidas reológicas}

Para determinação das propriedades reológicas, o cilindro interno foi acoplado ao equipamento e a amostra foi adicionada ao cilindro externo, conectado ao equipamento, após a estabilização da temperatura desejada. O cilindro externo foi totalmente preenchido com a amostra de sorvete. As medidas reológicas foram obtidas variando a taxa de deformação de 0,02 a $50 \mathrm{~s}^{-1}$ (curva ascendente) e de 50 a $0,02 \mathrm{~s}^{-1}$ (curva descendente), com um tempo total de ensaio de 4 minutos, com a tomada de 60 pontos para cada curva. Assim, obteve-se o reograma de cada amostra.

Os modelos testados foram: Bingham, Casson, Hersch-Bulkley e Lei da Potência (STEFFE, 1996). Porém, para obtenção dos parâmetros reológicos e para a determinação do seu comportamento, utilizou-se o modelo Lei da Potência (Equação 1), e a viscosidade para fluidos não newtonianos pela Equação 2.

$\tau=K \gamma^{\mathrm{n}}$

$\eta=K(\gamma)^{n-1}$

sendo:

$K=$ o índice de consistência $\left[\right.$ P.s $\left.{ }^{\mathrm{n}}\right]$;

$n=\mathrm{o}$ índice de comportamento [adimensional];

$\tau=$ a tensão de cisalhamento;

$\gamma=\mathrm{a}$ taxa de deformação $\left(\mathrm{s}^{-1}\right)$; e

$\eta=\mathrm{a}$ viscosidade (Pa.s).

A Equação 3 indica o modelo de Plástico de Bingham.

$\tau=\tau_{0}+\eta_{p l} \gamma$

sendo:

$\tau_{\mathrm{o}}=$ a tensão de cisalhamento inicial; e

$\eta_{\mathrm{pl}}=\mathrm{a}$ viscosidade plástica.

O modelo de Herschel-Bulkley é descrito na Equação 4:

$\tau=\tau_{o}+K(\lambda)^{n}$

E o modelo de Casson é apresentado através da Equação 5.

$\tau^{0,5}=\tau_{o}^{0,5}+K(\gamma)^{0,5}$

\section{Resultados e discussão}

\subsection{Composição dos diferentes tipos de sorvetes}

A Tabela 2 apresenta a composição centesimal dos sorvetes utilizados neste trabalho para $100 \mathrm{~g}$ de amostra.

\subsection{Caracterização reológica do sorvete}

\section{Comportamento do fluido}

Os reogramas dos diferentes tipos de sorvete estudados estão apresentados nas Figuras 2, 3 e 4, a temperaturas de -2, 0 e $2{ }^{\circ} \mathrm{C}$, respectivamente. Nos reogramas são apresentadas as curvas: ascendente e descendente de cada uma das amostras, sendo possível verificar se houve ou não o efeito de histerese.

Os reogramas mostram que todas as amostras apresentaram uma relação não linear entre a tensão de cisalhamento e a taxa de deformação nas temperaturas de $-2,0$ e $2{ }^{\circ} \mathrm{C}$. Portanto, seu comportamento é caracterizado como não newtoniano.

De acordo com a Figura 2 , na temperatura de $-2{ }^{\circ} \mathrm{C}$ observase que a amostra de limão, comparada com as demais, obteve maior tensão de cisalhamento, seguida da amostra de creme light. Este comportamento pode ser explicado pelo fato de ambas as amostras apresentarem maior fração de água e menor quantidade de gordura e, conseqüentemente, menor cremosi-

Tabela 2. Composição centesimal de diferentes tipos de sorvete.

\begin{tabular}{lrrrrr}
\hline \multirow{2}{*}{$\begin{array}{c}\text { Informação } \\
\text { nutricional** }\end{array}$} & \multicolumn{5}{c}{ Amostras } \\
\cline { 2 - 6 } & Creme & $\begin{array}{c}\text { Creme } \\
\text { light }\end{array}$ & Limão & $\begin{array}{c}\text { Soja } \\
\text { banana }\end{array}$ & Iogurte \\
\hline Carboidratos (g) & 22 & 8 & 16 & 31 & 23 \\
Proteínas (g) & 4 & 4 & 0 & 4 & 4 \\
Gorduras totais (g) & 7 & 0 & 3 & 2 & 2 \\
Gorduras saturadas (g) & 3 & 0 & 1 & 0 & 2 \\
Colesterol (mg) & 15 & 0 & 0 & 0 & 5 \\
Fibra alimentar (g) & 0 & 0 & 0 & ND $^{*}$ & 0 \\
Cálcio (mg) & 118 & 143 & 120 & 16 & 143 \\
Sódio (mg) & 53 & 60 & $<25$ & ND $^{*}$ & 60 \\
\hline *ND = não determinado; e**fornecido por Amoratto Sorvetes Artesanais.
\end{tabular}

${ }^{*} \mathrm{ND}=$ não determinado; $\mathrm{e}^{* *}$ fornecido por Amoratto Sorvetes Artesanais.

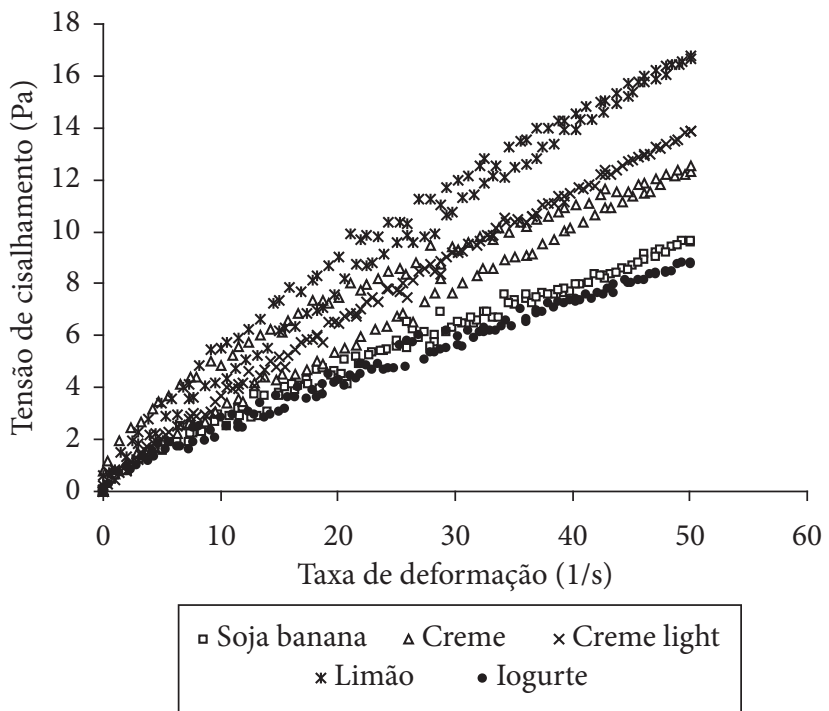

Figura 2. Reograma das amostras dos diferentes tipos de sorvete na temperatura de $-2{ }^{\circ} \mathrm{C}$. 


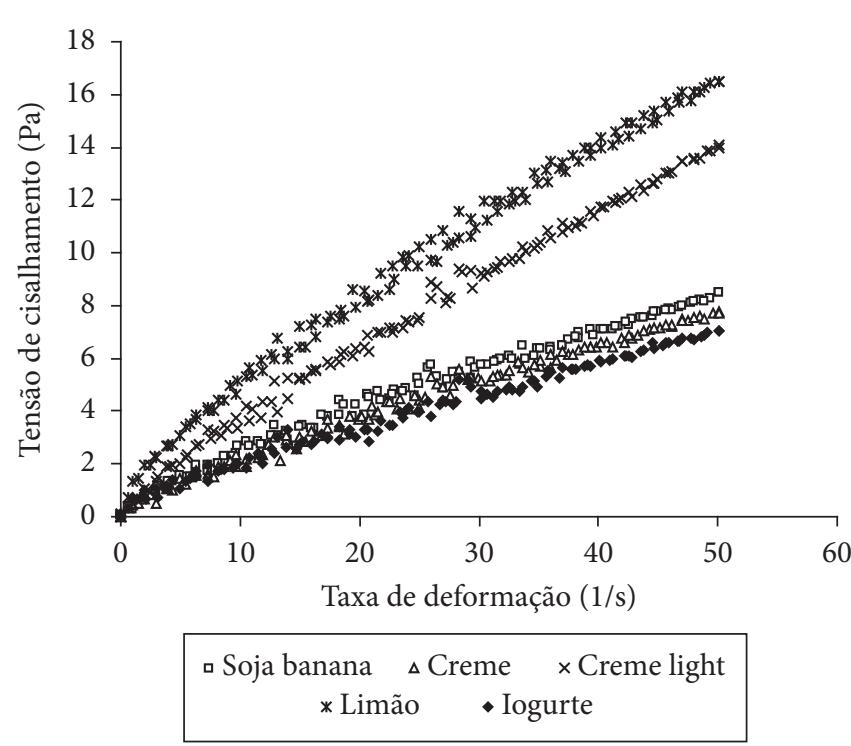

Figura 3. Reograma das amostras dos diferentes tipos de sorvete na temperatura de $0{ }^{\circ} \mathrm{C}$.

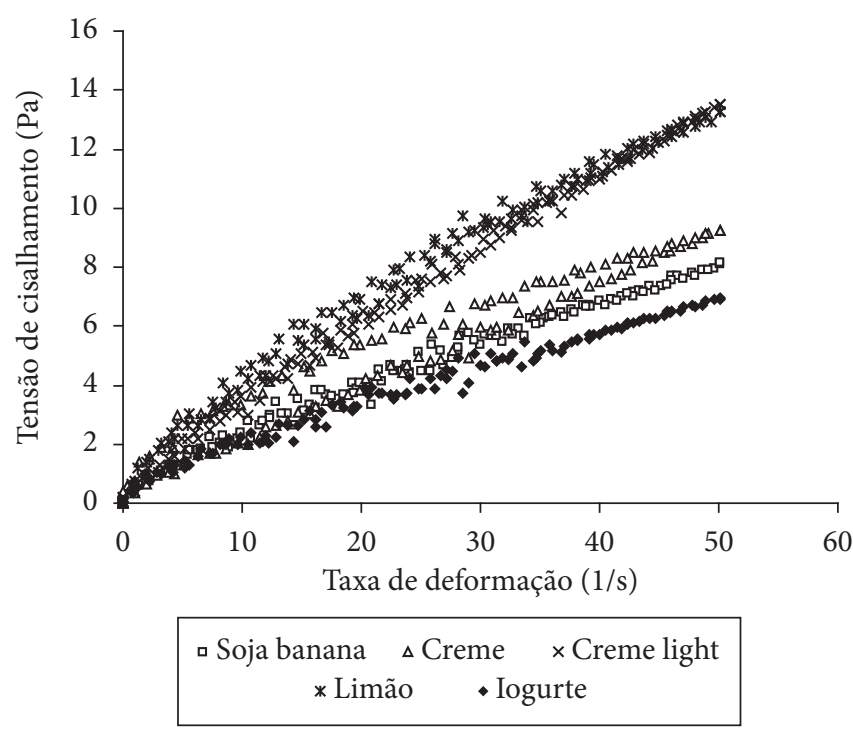

Figura 4. Reograma das amostras dos diferentes tipos de sorvete na temperatura de $2{ }^{\circ} \mathrm{C}$.

dade. As amostras de soja banana e de iogurte apresentaram menor tensão de cisalhamento e comportamentos similares (Figura 2), sendo influenciados possivelmente pela consistência cremosa em ambas as amostras. Duas amostras apresentaram efeito de histerese, a de creme e a de limão. Sendo que a amostra de creme apresentou um efeito de histerese maior (área entre a curva ascendente e descendente), possivelmente pelo fato de apresentar maior quantidade de gordura. Esse efeito confirma que o fluido é dependente do tempo e a área de histerese é uma indicação de comportamento tixotrópico (TÁRREGA; DURAN; COSTELL, 2004).
$\mathrm{Na}$ temperatura de $0{ }^{\circ} \mathrm{C}$, novamente as amostras de limão e creme light apresentaram os maiores valores de tensão de cisalhamento, como mostrado pela Figura 3. Sendo que os valores obtidos nessa temperatura foram bastante próximos aos obtidos a $-2{ }^{\circ} \mathrm{C}$. Esse comportamento mostra que a variação de temperatura, de -2 a $0{ }^{\circ} \mathrm{C}$, não é o fator principal que influencia o comportamento reológico das amostras de sorvete, para pequenos intervalos de temperatura, conforme os utilizados neste trabalho.

As amostras de soja banana, creme e iogurte obtiveram valores de tensão de cisalhamento próximos entre si e menores do que as amostras de creme light e limão. Comparando os resultados observa-se que, a $0{ }^{\circ} \mathrm{C}$ os valores de tensão de cisalhamento foram menores que a $-2{ }^{\circ} \mathrm{C}$. Valores de tensão de cisalhamento podem ser influenciados pela composição, principalmente a fração de carboidratos que é próxima para as três amostras que apresentaram resultados semelhantes. Entretanto, outros fatores como, pressão de homogeneização, incorporação de ar e agregação dos cristais de gelo podem afetar a tensão de cisalhamento (KUS; ALTAN; KAIA, 2005; SOFJAN; HARTEL, 2004) gerando resultados diferentes, conforme observado neste trabalho.

$\mathrm{O}$ efeito de histerese não foi observado à temperatura de $0{ }^{\circ} \mathrm{C}$. É praticamente inexistente a área entre a curva ascendente e a descendente para todas as amostras.

A Figura 4 mostra que o comportamento das amostras de sorvete ao longo das taxas de deformação foi semelhante àquele obtido nas temperaturas de -2 e $0{ }^{\circ} \mathrm{C}$. Novamente, as amostras que apresentaram maiores valores de tensão de cisalhamento foram a de limão e a de creme light.

As amostras de creme, soja banana e iogurte apresentaram novamente os menores valores de tensão de cisalhamento.

À temperatura de $2{ }^{\circ} \mathrm{C}$, o efeito de histerese foi observado novamente para as amostras de creme com comportamento semelhante ao obtido a $-2^{\circ} \mathrm{C}$, com menor área visual. Uma possível explicação estaria relacionada com a mudança estrutural das amostras (TÁRREGA; DURAN; COSTELL, 2004). Entretanto, a análise da estrutura não foi realizada neste trabalho, por isso não são possíveis conclusões mais detalhadas para o comportamento apresentado. De acordo com Halmos e Tiu (1981), a área entre a curva ascendente e a descendente é um índice de energia por unidade de tempo e volume, necessário para eliminar a influência do tempo no comportamento do fluido. Neste trabalho, a $0{ }^{\circ} \mathrm{C}$ o fluido não apresentou comportamento tixotrópico e muito provavelmente influenciou no comportamento das curvas. Kus, Altan e Kaya (2004) mostram diferentes efeitos de histerese para sorvete à base de leite, com 1,5\% de leite desnatado e açúcar, às temperaturas de $5 \mathrm{a} 50^{\circ} \mathrm{C}$, porém em alguns casos o efeito da histerese não foi significativo.

O valor negativo de algumas das tensões iniciais (Tabela 5) para o modelo de Herschel-Bulkley é o resultado do ajuste do próprio modelo influenciado por erros experimentais. As baixas taxas de cisalhamento provocam oscilações nas medidas, em reômetro de cilindros concêntricos, não permitindo a determinação precisa da tensão inicial. 


\section{Viscosidade}

O comportamento da viscosidade, em função da taxa de deformação das amostras às temperaturas de $-2,0$ e $2{ }^{\circ} \mathrm{C}$, está apresentado nas Figuras 5, 6 e 7, respectivamente.

Observa-se através das figuras que, a viscosidade de todas as amostras diminuiu com as taxas de deformação. Sendo que, as amostras de creme light e limão apresentaram as maiores viscosidades nas três temperaturas. E as amostras de iogurte, soja banana e creme apresentaram as menores viscosidades. O comportamento apresentado, possivelmente é função da estrutura do sorvete que, quando congelado apresenta diferença na formação de cristais de gelo entre as amostras com e sem gordura (SMITH; BAKSHI; LOMAURO, 1984). Neste caso, não houve influência da temperatura sobre a viscosidade, muito

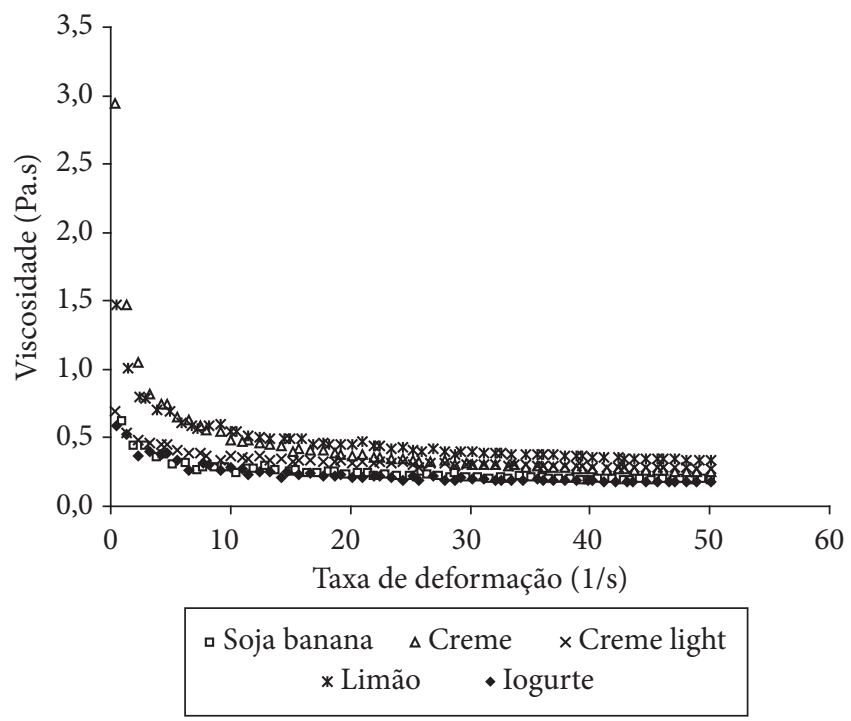

Figura 5. Viscosidade $x$ taxa de deformação das amostras dos diferentes tipos de sorvete na temperatura de $-2{ }^{\circ} \mathrm{C}$.

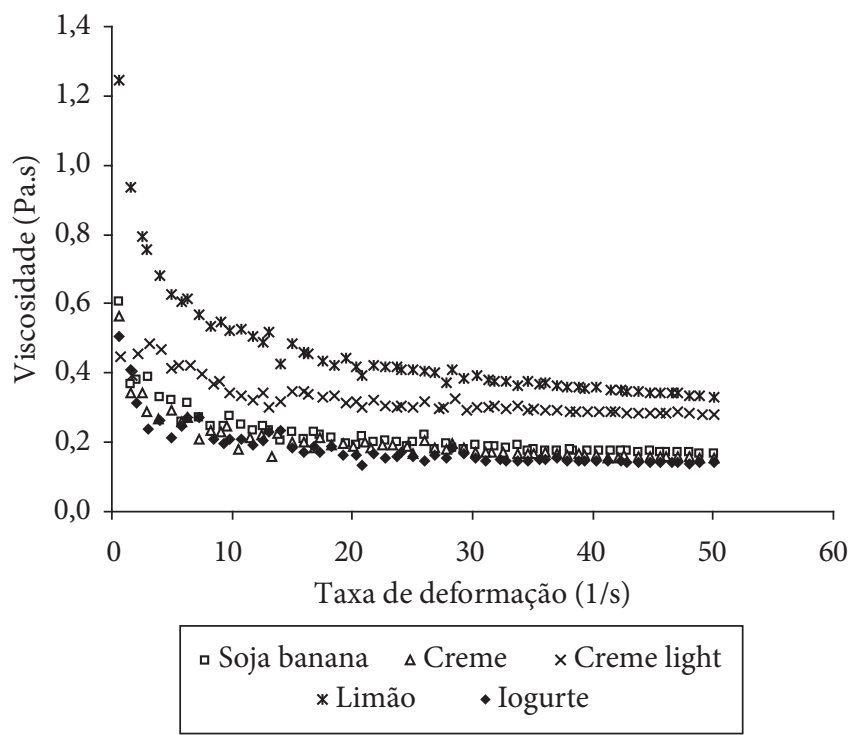

Figura 6. Viscosidade $\mathrm{x}$ taxa de deformação das amostras dos diferentes tipos de sorvete na temperatura de $0{ }^{\circ} \mathrm{C}$. provavelmente pelo pequeno intervalo estudado neste trabalho. Resultados semelhantes foram obtidos por Mendonça (2002) para o estudo reológico de iogurte light e integral.

A análise global dos reogramas e dos gráficos de viscosidade em função da taxa de deformação realizada anteriormente não permite caracterizar completamente o comportamento reológico de cada uma das amostras.

Para caracterização do comportamento reológico das amostras, quatro diferentes modelos foram testados: Bingham, Casson, Herschel-Bulkley e Lei da Potência. Sendo que, o modelo Lei da Potência apresentou o melhor ajuste aos resultados.

A Tabela 3 mostra os valores dos parâmetros reológicos obtidos para cada amostra, em cada uma das temperaturas estudadas, através do ajuste do modelo Lei da Potência.

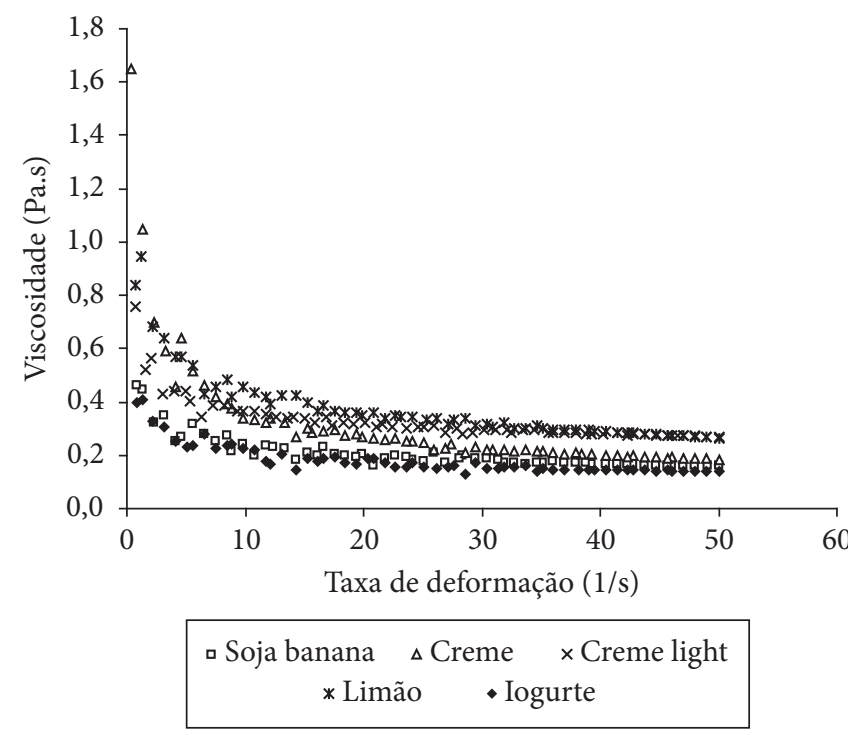

Figura 7. Viscosidade $\mathrm{x}$ taxa de deformação das amostras dos diferentes tipos de sorvete à temperatura de $2{ }^{\circ} \mathrm{C}$.

Tabela 3. Parâmetros reológicos obtidos pelo modelo Lei da Potência das diferentes amostras de sorvete.

\begin{tabular}{lcccc}
\hline Amostras & Parâmetros & \multicolumn{3}{c}{ Temperaturas $\left({ }^{\circ} \mathrm{C}\right)$} \\
\cline { 3 - 5 } & & -2 & 0 & 2 \\
\hline Creme & $K$ & 1,1678 & 1,5376 & 0,9942 \\
& $n$ & 0,5530 & 0,7777 & 0,5663 \\
Creme light & $r$ & 0,9985 & 0,9927 & 0,9913 \\
& $K$ & 0,7716 & 0,7696 & 0,7683 \\
& $n$ & 0,8238 & 0,8239 & 0,8193 \\
Limão & $r$ & 0,9988 & 0,9974 & 0,9987 \\
& $K$ & 1,0320 & 0,9993 & 0,9436 \\
Soja banana & $n$ & 0,7054 & 0,7196 & 0,7003 \\
& $K$ & 0,9989 & 0,9987 & 0,9977 \\
& $n$ & 0,7393 & 0,7195 & 0,6819 \\
Iogurte & $n$ & 0,7454 & 0,7321 & 0,7543 \\
& $r$ & 0,9930 & 0,9956 & 0,9924 \\
& $K$ & 0,7615 & 0,6469 & 0,6665 \\
& $n$ & 0,7087 & 0,7491 & 0,7271 \\
& $r$ & 0,9930 & 0,9993 & 0,9896 \\
\hline
\end{tabular}


Os valores do coeficiente de correlação $r$ demonstram que o modelo Lei da Potência foi adequado para o ajuste dos dados de tensão de cisalhamento e para a taxa de deformação dos diferentes tipos de sorvete, nas três temperaturas ensaiadas. De um modo geral, os sorvetes apresentaram valores de coeficiente de correlação $r$ iguais a 0,99 . Com exceção da amostra de iogurte, na temperatura de $2{ }^{\circ} \mathrm{C}$, que apresentou o coeficiente $r$ igual a 0,9896 .

As propriedades reológicas da maioria dos sorvetes têm sido descritas como pseudoplásticas (COTREL; GEOFREY; PHILLIPS, 1980; GOFF; DAVIDSON, 1992). Como esperado, os diferentes tipos de sorvete avaliados apresentaram comportamento não newtoniano nas três temperaturas de estudo. Os índices de comportamento $(n$ ) obtidos variaram de 0,5530 (menor valor) a 0,8239 (maior). Foram menores que a unidade, portanto, as amostras estudadas podem ser caracterizadas como pseudoplásticas. Ou seja, a viscosidade diminui com a taxa de deformação.

As amostras de creme light, limão e soja banana apresentaram pequena variação no valor de $n$, nas diferentes temperaturas. Para as amostras de creme e iogurte houve uma variação um pouco maior no valor do índice de comportamento do escoamento $(n)$. No entanto, os valores dos índices de comportamento do escoamento $(n)$ das amostras nas três temperaturas $\left(-2,0\right.$ e $\left.2{ }^{\circ} \mathrm{C}\right)$ mostram que não houve mudança no comportamento reológico. Todas as amostras apresentaram comportamento pseudoplástico nas temperaturas avaliadas. A Tabela 4 apresenta os valores do coeficiente de correlação $r$ dos demais modelos que o software permitiu ajustar, para cada uma das amostras, nas distintas temperaturas.

Os resultados encontrados neste trabalho estão de acordo com estudos realizados por Kaya e Tekin (2001). Os autores avaliaram o efeito de diferentes concentrações de um estabilizante natural na reologia de sorvetes e encontraram também

Tabela 4. Valores dos coeficientes de correlação $r$ dos modelos de Bingham, Casson e Herschel-Bulkley das diferentes amostras de sorvete.

\begin{tabular}{llccc}
\hline \multirow{2}{*}{ Amostras } & \multicolumn{1}{c}{ Modelos } & \multicolumn{3}{c}{ Temperaturas $\left({ }^{\circ} \mathrm{C}\right)$} \\
\cline { 3 - 5 } & & -2 & 0 & 2 \\
\hline Creme & Bingham & 0,9836 & 0,9915 & 0,9850 \\
& Herschel-Bulkley & 0,9988 & 0,9959 & 0,9968 \\
& Casson & 0,9960 & 0,9949 & 0,9951 \\
Creme light & Bingham & 0,9974 & 0,9978 & 0,9978 \\
& Herschel-Bulkley & - & 0,9990 & 0,9993 \\
& Casson & 0,9990 & 0,9989 & 0,9991 \\
Limão & Bingham & 0,9913 & 0,9931 & 0,9903 \\
& Herschel-Bulkley & 0,9992 & 0,9990 & 0,9988 \\
\multirow{5}{*}{ Soja banana } & Casson & 0,9974 & 0,9979 & 0,9967 \\
& Bingham & 0,9952 & 0,9936 & 0,9925 \\
& Herschel-Bulkley & 0,9971 & 0,9973 & 0,9960 \\
Iogurte & Casson & 0,9971 & 0,9969 & 0,9955 \\
& Bingham & 0,9927 & 0,9906 & 0,9904 \\
& Herschel-Bulkley & 0,9965 & 0,9934 & 0,9938 \\
& Casson & 0,9962 & 0,9933 & 0,9936 \\
\hline
\end{tabular}

comportamento pseudoplástico para as amostras estudadas. $\mathrm{O}$ índice de comportamento do escoamento $(n)$ obtido variou de 0,77 a 0,96 para amostras de sorvetes à base de leite, nas temperaturas de $10,20,30,40$ e $50{ }^{\circ} \mathrm{C}$. Os mesmos autores citam que, Goff e Davidson (1992), ao pesquisarem a reologia de sorvetes, encontraram valores de índice de comportamento do escoamento em torno de 0,7. Goff e Davidson (1992), ao estudarem o efeito de estabilizantes no comportamento reológico de sorvete, encontraram valores de índice de comportamento do escoamento na faixa de 0,98 a 0,68 , usando como estabilizante a goma guar, e 0,88 a 0,48 para o estabilizante goma locusta. Aime et al. (2001), ao avaliarem o comportamento reológico de sorvetes de creme com diferentes quantidades de gordura, encontraram valores de $n$ iguais a: 0,661 (sorvete com $0,4 \%$ de gordura); 0,430 (sorvete com 2,5\% de gordura); 0,370 (sorvete com $5 \%$ de gordura); e 0,433 (sorvete com $10 \%$ de gordura). A temperatura utilizada pelos autores nos ensaios reológicos foi de $30^{\circ} \mathrm{C}$. Rao e Anantheswaran (1982) encontraram valores de $n$ entre 0,48 a 0,55 .

Os parâmetros reológicos obtidos com o ajuste dos dados aos modelos de Bingham, Casson e Herschel-Bulkley estão apresentados na Tabela 5.

O comportamento da viscosidade observada no presente trabalho pode ter sido influenciado pelo tratamento térmico da mistura como as transições de fases, fusão dos lipídios e cristalização da água durante o batimento. Segundo Varnam e Sutherland (1994), a viscosidade da calda aumenta como resultado da interação entre o estabilizante e outros solutos, como os açúcares, o que também pode ter influenciado os resultados deste trabalho.

\section{Conclusões}

As diferentes amostras de sorvete estudadas neste trabalho apresentaram comportamento não newtoniano, nas temperaturas de $-2,0$ e $2{ }^{\circ} \mathrm{C}$. Os modelos reológicos de Bingham, Casson, Herschel-Bulkley e Lei da Potência, utilizados para o ajuste dos dados experimentais, mostraram-se adequados, com coeficientes de correlação superiores a 0,98 para todas as amostras, nas três diferentes temperaturas de ensaio. Os parâmetros reológicos foram obtidos para todos os modelos, sendo que o modelo da Lei da Potência apresentou os melhores resultados $(R=0,99)$. $\mathrm{O}$ efeito de histerese foi observado para as amostras de creme e limão nas temperaturas de $-2 \mathrm{e} 2{ }^{\circ} \mathrm{C}$, mostrando a dependência do fluido com o tempo. As amostras apresentaram valores de índice de comportamento $(n)$ inferiores a um, caracterizando o comportamento pseudoplástico, visto que a viscosidade das amostras diminuiu com as taxas de deformação aplicadas. E, em alguns casos, como os das amostras de creme e limão, observouse que as mesmas apresentaram comportamento tixotrópico.

\section{Agradecimentos}

À Empresa Amoratto Sorvetes Artesanais, pelos recursos financeiros. Ao Laboratório de Materiais (LABMAT), pela disponibilidade do reômetro para a realização dos ensaios reológicos. 
Tabela 5. Parâmetros reológicos dos modelos de Bingham, Casson e Herschel-Bulkley das diferentes amostras de sorvete.

\begin{tabular}{|c|c|c|c|c|c|c|}
\hline \multirow[t]{3}{*}{ Amostras } & \multirow{3}{*}{$\begin{array}{c}\text { Temperatura } \\
\left({ }^{\circ} \mathrm{C}\right)\end{array}$} & \multicolumn{5}{|c|}{ Parâmetros dos modelos } \\
\hline & & \multicolumn{2}{|c|}{ Bingham } & \multirow{2}{*}{$\begin{array}{l}\text { Casson } \\
\tau_{0}(\mathrm{~Pa})\end{array}$} & \multicolumn{2}{|c|}{ Herschel-Bulkley } \\
\hline & & $\tau_{0}(P a)$ & $\eta_{p l}(P a . s)$ & & $\tau_{0}(\mathrm{~Pa})$ & $n$ \\
\hline \multirow[t]{3}{*}{ Creme } & -2 & 2,7490 & 0,2097 & 1,3580 & $-0,05138$ & 0,6917 \\
\hline & 0 & 0,6982 & 0,1472 & 0,1975 & $-0,0273$ & 0,7620 \\
\hline & 2 & 1,8270 & 0,1575 & 0,8516 & 0,3823 & 0,6289 \\
\hline \multirow[t]{3}{*}{ Creme light } & -2 & 0,8558 & 0,2686 & 0,1819 & - & - \\
\hline & 0 & 0,7520 & 0,2715 & 0,1382 & 0,1033 & 0,8694 \\
\hline & 2 & 0,9099 & 0,2591 & 0,1978 & 0,1948 & 0,8517 \\
\hline \multirow[t]{3}{*}{ Limão } & -2 & 2,135 & 0,3118 & 0,7612 & $-0,0514$ & 0,6917 \\
\hline & 0 & 1,8970 & 0,3110 & 0,6249 & 0,03385 & 0,7250 \\
\hline & 2 & 1,7340 & 0,2484 & 0,6258 & $-0,1092$ & 0,6798 \\
\hline \multirow[t]{3}{*}{ Soja banana } & -2 & 0,8281 & 0,1785 & 0,2171 & 0,2422 & 0,8289 \\
\hline & 0 & 0,8323 & 0,1564 & 0,2472 & 0,1190 & 0,7759 \\
\hline & 2 & 0,7523 & 0,1506 & 0,2143 & 0,0842 & 0,7813 \\
\hline \multirow[t]{3}{*}{ Iogurte } & -2 & 0,9386 & 0,1625 & 0,2927 & 0,1874 & 0,7734 \\
\hline & 0 & 0,6975 & 0,1294 & 0,2048 & 0,1859 & 0,8001 \\
\hline & 2 & 0,7160 & 0,1279 & 0,2172 & 0,1434 & 0,7798 \\
\hline
\end{tabular}

\section{Referências bibliográficas}

AIME, D. B. et al. Textural analysis of fat reduced vanilla ice cream products. Food Research International, v. 34, p. 237-246, 2001.

ARBUCKLE, W. S. Ice cream. 3 ed. USA: AVI Publishing Company, 1977.517 p.

COTRELL, J. I. L.; GEOFREY, P.; PHILIPS, G. O. The effect of stabilizers on the viscosity of an ice-cream mix. Journal of the Science of Food and Agriculture, v. 31, p. 1066-1070, 1980.

DICKINSON, E.; STAINSBY, G. Colloids in Foods. London: Applied Science Publishers, 1982. p. 382-383.

GOFF, H. D.; DAVIDSON, V. J. Flow characteristics and holding time calculations of ice cream mixes in HTST holding tubes. Journal of Food Protection, v. 55, p. 34-37, 1992.

HOLDSWORTH, S. D. Applicability of rheological models to the interpretation of flow and processing behaviour of fluid food products. Journal of Texture Studies, v. 2, n. 4, p. 393-418, 1971.

HALMOS, A. L.; TIU, C. Liquid foodstuff exhibiting yields stress and shear degradability. Journal of Texture Studies, v. 12, p. 39-46, 1981.

KAYA, S.; TEKIN, A. R. The effect of salep content on the rheological characteristics of a typical ice-cream mix. Journal of Food Engineering, v. 47, p. 59-62, 2001.

KUS, S.; ALTAN, A.; KAYA, A. Rheological behavior and timedependent characterization of ice cream mix with different salep content. Journal of Texture Studies, v. 36, p. 273-288, 2005.

MENDONÇA, E. Estudo do comportamento reológico de iogurtes comerciais. Florianópolis, 2002, 63p. Dissertação - (Mestrado em Engenharia de Alimentos), Universidade Federal de Santa Catarina - UFSC.

INSTITUTO ADOLFO LUTZ. NORMAS DO INTITUTO ADOLFO LUTZ. Métodos químicos e físicos de análise de alimentos. 2 ed. São Paulo, 1976.

RAO, M. A.; ANANTHESWARAN, R. C. Rheology of fluids in food processing. Food Technology, v. 36, n. 2, p. 116-126, 1982.

SMITH, D. E.; BAKSHI, A. S.; LOMAURO, C. J. Changes in freezing point and rheological properties of ice cream mix as a function of sweetner system and whey substitution. Milchwissenschaft, v. 39, p. 455, 1984.

SOFJAN, R. P.; HARTEL, R. W. Effects of overrun on structural and physical characteristics of ice cream. International Dairy Journal, v. 14, p. 255-262, 2004.

STEFFE, J. F. Rheological methods in food process engineering. 2 ed. Miami: Freeman Press, 1996.

TABILO-MUNIZAGA, G.; BARBOSA-CÁNOVAS, G. V. Rheology for the food industry. Journal of Food Engineering, v. 67, p. 147-156, 2005.

TÁRREGA, A.; DURÁN, L.; COSTELL, E. Flow behavior of semi-solid dairy deserts. Effect of temperature. International Dairy Journal, v. 14, p. 345-353, 2004.

VARNAM, A. H.; SUTHERLAND, J. P. Milk and milk products: technoloby, chemistry and microbiology. 1 ed. Canadá: An Aspen Publication, 1994. 464 p. (Food products series, vol. 1.)

VASQUES, C. T. Reologia do suco de goiaba: efeito da diluição e do tamanho de partícula. Florianópolis, 2003. 66p. Dissertação - (Mestrado em Engenharia de Alimentos), Universidade Federal de Santa Catarina - UFSC. 